\title{
Nota sobre culicídeos adultos coletados na região sul do Brasil
}

\author{
Adult culicidae captured in a rural area in southern Brazil \\ Orlando Carlos Barbosa", Ueslei Teodoro"*, Ana Leuch Lozovei"**, Vicente La Salvia Filho**, \\ Roberto Palma Spinosa*, Edson Maurício de Lima**, Maria Eugênia Moreira Costa Ferreira****
}

\begin{abstract}
BARBOSA, O.C. et al. Nota sobre culicídeos adultos coletados na região sul do Brasil. Rev. Saúde Pública, 27: 214-6, 1993. São apresentados os resultados obtidos de coletas de mosquitos, com armadilha luminosa de Shannon, às margens de uma mata modificada, e armadilhas de Falcão na mesma mata e em ecótopos extra-florestais, no Município de Terra Boa, Estado do Paraná, Brasil, de setembro de 1988 a abril de 1990. Verificou-se a prevalência das espécies de culicídeos e compararam-se os dois métodos de coletas.
\end{abstract}

Descritores: Mosquitos. Ecologia de vetores. Insetos vetores.

A associação de mosquitos com doenças humanas como a malária, febre amarela, dengue e outras, tem estimulado investigaçōes sobre a distribuição geográfica e de diversos aspectos do comportamento das espécies que compoem a fauna desses dípteros da familia Culicidae.

No Estado do Paraná somente as pesquisas mais recentes englobam a fauna de culicídeos como um todo ${ }^{1,5,6}$. Com o objetivo de ampliar o conhecimento sobre esses dípteros, nesse Estado, pesquisou-se a composição da fauna e a prevalência das espécies, e compararam-se dois métodos de coletas, numa área ainda não investigada.

Os mosquitos foram capturados de setembro de 1988 a abril de 1990, na fazenda Palmital, propriedade da Companhia Melhoramentos Norte do Paraná, no Município de Terra Boa, na região Norte do Paraná. Durante 20 meses efetuaram-se 20 coletas, sempre por duas pessoas, uma vez por mês, empregando-se armadilha luminosa de Shannon, às margens de uma mata modificada, das 18 às $06 \mathrm{~h}$. Com 10 armadilhas de Falcão ${ }^{2}$ (1981) foram feitas 40 coletas em ecótopos florestal e extraflorestal (domicílio e peridomicílio), duas vezes por mês, das 21 às 03 horas, dando 240 horas por armadilha.

\footnotetext{
* Fundação Nacional de Saúde - Distrito de Londrina Londrina, PR - Brasil

* Departamento di: Análises Clínicas da Universidade Estadual de Maringá - Maringá, PR - Brasil

*** Departamento de Patologia Básica da Universidade Federal do Paraná, Centro Politécnico - Paraná - Brasil

**** Departamento de Geografia da Universidade Estadual de Maringá - Maringá, PR - Brasil
}

Separatas/Reprints: O.C. Barbosa - Av. Centenário, 55 - Aeroporto - 87050-040 - Maringá, PR - Brasil
Com a armadilha de Shannon coletaram-se 2.996 mosquitos, e em armadilhas de Falcão, 1.726. Com estas últimas, coletaram-se 1.171 mosquitos nos ecótopos florestais e 555 nos extraflorestais (Tabela).

É evidente a prevalência de Chagasia fajardoi, Aedes scapularis, Culex quinquefasciatus, Coquillettidea venezuelensis, Culex coronator e Culex mollis, pois estas espécies, em conjunto, representaram $61,1 \%$ dos 4.722 insetos identificados ( Tabela).

O encontro de Aedes scapularis, Culex coronator e Culex mollis, na localidade onde se executou o presente trabalho, induz à especulação de que essas espécies podem estar adaptando-se aos ambientes antropogênicos, pois estes mosquitos já foram assinalados nesses ambientes, no Estado do Paraná1,6,7.

Forattini e col. ${ }^{3}$ (1978) sugeriram uma possivel associação entre o vírus da encefalite e o comportamento de Aedes scapularis em ambiente alterado, no Estado de São Paulo, e Forattini \& Gomes ${ }^{4}$ (1988), devido a presença de Haemagogus leucocelaenus nas proximidades da cidade de Araraquara, onde estava presente Aedes aegypti, comentaram da possibilidade de ocorrência da febre amarela urbana. Ressalta-se que Aedes scapularis e Haemagogus leucocelaenus estão sendo assinalados na presente nota, e Aedes aegypti, tem sido notificado em vários municípios do Estado do Paraná, nas rotinas da Fundação Nacional de Saúde para a localização e controle dos criadouros desse mosquito.

As informaçס̃es obtidas na presente nota permitem concluir que: 1) na armadilha de Shannon obteve-se melhor rendimento nas coletas do que no conjunto das armadilhas de Falcão; 2) existe potencial de transmissão de arbovirus urbanos no Estado 
Tabela. Culicideos coletados com armadilhas de Shannon e Falcão, na fazenda Palmital, Município de Terra Boa, Estado do Paraná, Brasil, de setembro de 1988 a abril de 1990.

\begin{tabular}{|c|c|c|c|c|c|}
\hline Espécie local & SF & FF & FE & Total & $\%$ \\
\hline $\begin{array}{l}\text { Anopheles argyritarsis } \\
\text { Anopheles evansae } \\
\text { Anopheles fluminensis } \\
\text { Anopheles galvaoi } \\
\text { Anopheles intermedius } \\
\text { Anopheles lutzi } \\
\text { Anopheles parvus } \\
\text { Anopheles triannulatus } \\
\text { An. (Nyssorhynchus) sp } \\
\text { Anopheles spp } \\
\text { Aedes scapularis } \\
\text { Aedes serratus } \\
\text { Aedes stigmaticus } \\
\text { Aedes terrens } \\
\text { Aedes sp } \\
\text { Aedomyia squamipennis } \\
\text { Chagasia fajardoi } \\
\text { Coquillettidea venezuelensis } \\
\text { Culex acharistus } \\
\text { Culex bonneae } \\
\text { Culex chidesteri } \\
\text { Culex corniger } \\
\text { Culex coronator } \\
\text { Culex declarator } \\
\text { Culex dolosus } \\
\text { Culexmollis } \\
\text { Culex quinquefasciatus } \\
\text { Culex (Culex) spp } \\
\text { Culex (Lutzia) spp } \\
\text { Culex (Melanoconium) spp } \\
\text { Culex spp } \\
\text { Haemagogus leucocelaenus } \\
\text { Mansonia titilans } \\
\text { Psorophora cingulata } \\
\text { Psorophora ferox } \\
\text { Psorophora varipes } \\
\text { Psorophora sp } \\
\text { Sabethes (Sabethoides) sp } \\
\text { Uranotaenia spp } \\
\text { (n) }\end{array}$ & $\begin{array}{r}28 \\
50 \\
64 \\
- \\
2 \\
78 \\
9 \\
69 \\
- \\
11 \\
389 \\
17 \\
2 \\
1 \\
- \\
- \\
1.382 \\
190 \\
2 \\
- \\
- \\
- \\
65 \\
14 \\
11 \\
52 \\
50 \\
68 \\
6 \\
118 \\
235 \\
14 \\
60 \\
2 \\
2 \\
1 \\
- \\
1 \\
3\end{array}$ & $\begin{array}{r}1 \\
18 \\
18 \\
1 \\
- \\
21 \\
11 \\
52 \\
6 \\
3 \\
21 \\
13 \\
- \\
1 \\
1 \\
1 \\
154 \\
16 \\
27 \\
1 \\
1 \\
\overline{84} \\
38 \\
42 \\
107 \\
62 \\
30 \\
\overline{18} \\
275 \\
- \\
-7 \\
- \\
-1 \\
\overline{1} \\
40\end{array}$ & $\begin{array}{r}2 \\
22 \\
3 \\
4 \\
- \\
1 \\
- \\
14 \\
- \\
1 \\
26 \\
1 \\
- \\
- \\
- \\
1 \\
11 \\
22 \\
- \\
- \\
4 \\
5 \\
54 \\
6 \\
3 \\
22 \\
179 \\
3 \\
2 \\
59 \\
89 \\
- \\
16 \\
- \\
- \\
- \\
- \\
5\end{array}$ & $\begin{array}{r}31 \\
90 \\
85 \\
5 \\
2 \\
100 \\
20 \\
135 \\
6 \\
15 \\
436 \\
31 \\
2 \\
2 \\
1 \\
2 \\
1.547 \\
228 \\
29 \\
1 \\
5 \\
5 \\
203 \\
58 \\
56 \\
181 \\
291 \\
101 \\
8 \\
295 \\
599 \\
14 \\
83 \\
2 \\
2 \\
1 \\
1 \\
1 \\
1 \\
48\end{array}$ & $\begin{array}{r}0,65 \\
1,90 \\
1,79 \\
0,10 \\
0,04 \\
2,11 \\
0,42 \\
2,85 \\
0,12 \\
0,31 \\
9,23 \\
0,65 \\
0,04 \\
0,04 \\
0,02 \\
0,04 \\
32,75 \\
4,82 \\
0,61 \\
0,02 \\
0,10 \\
0,10 \\
4,30 \\
1,22 \\
1,18 \\
3,83 \\
6,25 \\
2,13 \\
0,16 \\
6,24 \\
12,78 \\
0,29 \\
1,75 \\
0,04 \\
0,04 \\
0,02 \\
0,02 \\
0,02 \\
1,01\end{array}$ \\
\hline Total & 2.996 & 1.171 & 555 & 4.722 & 100,0 \\
\hline
\end{tabular}

SF - Armadilha de Shannon no ambiente florestal.

FF - Armadilhas de Faleão no ambiente florestal.

FE - Armadilhas de Falcão no ambiente extra-florestal.

do Paraná face à existência de mosquitos a eles associados; 3) a armadilha de Falcão não deve ser usada para coletas de culicídeos, pois os insetos sofrem intensa descamação, dificultando a identificação.

\section{Agradecimentos}

À Companhia Melhoramentos Norte do Paraná de Cianorte, pelo auxílio proporcionado para a realização do presente trabalho.

BARBOSA, O. C. et al. [Adult culicidae captured in a rural area in Southem Brazil]. Rev. Saúde Pública, 27:
214-6, 1993. During the period from September 1988 to April 1990, mosquitoes were captured using Shannon light trap and Falcão light traps, in Terra Bo? county, in the north of Paraná State, Brazil. The Shannon trap was installed in the outlying modified forest and Falcão traps were installed in the modified forest and domiciliary areas. The prevalence of species was verified and the methods of capture compared.

Keywords: Mosquitoes. Ecology, vectors. Insect vectors.

\section{Referênclas Bibliográficas}

1. CONSOLIM, J.; PELLEGRINT, N.J. de M.; LUZ, E. Culicídeos das áreas de Itaipu, Estado do Paraná, Brasil. 
Município de Foz do Iguaçu (Diptera - Culicidae) Acta. Biol. Paranaense [no prelo].

2. FALCÃO, A.R. Um novo modelo de amadilha luminosa de sucçẫo para pequenos insetos. Mem. Inst. Oswaldo Cruz, 76: 303-5, 1981.

3. FORATTINI, O.P.; GOMES, A. de C.; GALATI, E.A.B.; RABELLO, E.X.; IVERSSON, L.B. Estudos ecológicos sobre mosquitos Culicidae no Sistema da Serra do Mar, Brasil. Rev. Saúde Pública, 12: 297-25, 1978.

4. FORATTINI, O.P. \& GOMES, A. de C. Biting activity of Aedes scapularis (Rondoni) and Haemagogus mosquitoes in Southem Brazil (Diptera: Culicidae). Rev. Sauide Pública, 22: 84-93, 1988.

5. FUNDAÇÃO NACIONAL DE SAUDE - MINISTÉRIO DA SAÚDE. Programa de controle sanitário da usina hidrelétrica de Taquaruçu, relatórios mensais de entomologia. Curitiba, $1991(04 / 91,06 / 91,08 / 91)$.

6. LOPES, J. Ecologia de mosquitos (Diptera: Culicidae) que procriam em criadouros naturais e artificiais em área rural, Londrina e Cambé, Paraná, Brasil. Curitiba, 1992. [Tese de Doutorado - Universidade Federal do Paraná].

7. SILVA, M.A.N. \& LOPES, J. Dados sobre a potencialidade criadoura de Culicidae (Diptera) do cemitétio São Pedro, Londrina. Semina, 6: 133-39, 1985.

Recebido para publicação em 1.12.1992

Reapresentado em 26.4.1993 Aprovado para publicação em 3.5.1993 